\title{
Nanoscale
}

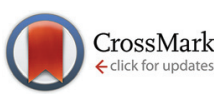

Cite this: Nanoscale, 2015, 7, 4514

Received 9th December 2014 Accepted 2nd February 2015

DOI: $10.1039 / c 4 n r 07277 d$

www.rsc.org/nanoscale

\section{Optimizing the oxygen evolution reaction for electrochemical water oxidation by tuning solvent properties}

\author{
Alessandro Fortunelli, ${ }^{\mathrm{a}, \mathrm{b}}$ William A. Goddard III, ${ }^{\mathrm{b}}$ Luca Sementa $^{\mathrm{a}}$ and \\ Giovanni Barcaro ${ }^{a}$
}

Electrochemical water-based energy cycles provide a most promising alternative to fossil-fuel sources of energy. However, current electrocatalysts are not adequate (high overpotential, lack of selectivity toward $\mathrm{O}_{2}$ production, catalyst degradation). We propose here mechanistic guidelines for experimental examination of modified catalysts based on the dependence of kinetic rates on the solvent dielectric constant. To illustrate the procedure we consider the fcc(111) platinum surface and show that the individual steps for the oxygen evolution reaction (OER) and oxygen reduction reaction (ORR) change systematically with the polarizability of the medium. Thus changing this environmental variable can be used to tune the rate determining steps and the barriers, providing a means for screening and validating new systems to optimize the rate determining steps for the ORR and OER reaction pathways.

\section{Introduction}

An electrochemical water-based energy cycle represents a promising means toward using dihydrogen as the energy carrier for an attractive sustainable alternative to fossil-fuel energy sources. ${ }^{1}$ In this strategy, sunlight excites electron-hole pairs that separate to electrodes that carry out the hydrogen evolution reaction (HER) and the oxygen evolution reaction (OER), i.e., water splitting, with the dihydrogen being used later via controlled water formation - the oxygen reduction reaction (ORR) and the hydrogen oxidation reaction (HOR) - in hydrogen fuel cells ${ }^{2}$ :

$$
\begin{aligned}
& \text { Water splitting (OER, HER): } \mathrm{H}_{2} \mathrm{O} \rightarrow \frac{1}{2} \mathrm{O}_{2}+\mathrm{H}_{2} \\
& \text { Water formation (ORR, HOR): } \frac{1}{2} \mathrm{O}_{2}+\mathrm{H}_{2} \rightarrow \mathrm{H}_{2} \mathrm{O}
\end{aligned}
$$

However, even the best catalyst electrodes exhibit slow process kinetics and large over-potentials in the OER and ORR processes, hampering the viability of this approach. Experimental optimization of these catalysts has proceeded

\footnotetext{
${ }^{a}$ CNR-ICCOM and IPCF, Consiglio Nazionale delle Ricerche, via Giuseppe Moruzzi 1, 56124 Pisa, Italy

${ }^{b}$ Materials and Process Simulation Center, California Institute of Technology, MC 139-74, Pasadena, California 91125, USA. E-mail: afloer@caltech.edu,

alessandro.fortunelli@cnr.it,wag@wag.caltech.edu
}

slowly. Quantum Mechanics (QM) calculations have provided new insights into the critical steps and how they depend on alloying, but the extrapolation to develop new catalysts and solvent conditions is not obvious. ${ }^{3-12}$ Even so, determining the atomistic mechanisms underlying the electrochemical transformations should provide guidance to achieve a significant acceleration in making the water energy cycle (1) and (2) efficient and competitive with respect to traditional carbonbased approaches. ${ }^{13}$

Unfortunately, in situ experimental characterization of the mechanisms, including the state of the interfaces, reactants, intermediates, reaction paths, and energy barriers under realistic operating reaction conditions is extremely difficult because of the transient character of the species involved and the difficulty in measuring atomistic details. ${ }^{14}$ We propose here a simple approach to obtain the mechanistic insight that can be used to suggest new designs for improved catalysts for experimental and computational testing. We illustrate this process using the fcc(111) platinum surface as a model catalyst. Here we consider the dependence of kinetic rates for all mechanistic steps as a function of the solvent dielectric constant, showing how this can provide mechanistic hypotheses. We show how this can be used to screen and validate proposed reaction paths, providing an effective tool to derive unique mechanistic understanding from experiment. This approach is particularly effective for the water oxidation/formation cycle because of the wide range of polarity changes in individual OER/ORR mechanistic steps. 
This article is organized as follows. Section 2 provides information on computational details, section 3 presents Results and discussion, while section 4 summarizes our conclusions.

\section{Computational approach}

The numerical results used here are taken from our previous work: ${ }^{7,15,16}$ here we recall the method and computational details. The methodology and computational details are as follows. The Perdew, Burke and Ernzerhof $(\mathrm{PBE})^{17}$ flavor of density-functional theory (DFT) was employed. We used SeqQuest $^{18}$ periodic DFT code in which Gaussian basis sets are used to describe the single-electron wave functions in place of the plane wave basis set used in many periodic DFT codes. The Gaussian basis set consisted of an optimized double- $\zeta$ plus polarization Gaussian-type basis set contracted from calculations on the most stable unit cell of the pure elements. Angular-momentum-projected norm-conserving nonlocal effective core potentials ${ }^{19-22}$ were used to replace the core electrons. Thus, the Pt atom is described with 16 explicit electrons ( $\operatorname{six} 5 \mathrm{p}$, one $6 \mathrm{~s}$, and nine $5 \mathrm{~d}$ in the ground state).

The real space grid density is 5 points $\AA^{-1}$, while the reciprocal space grid is $(5 \times 5 \times 0)$ for slab calculations. All calculations allowed the up spin-orbitals to be optimized independently of the down spin-orbitals (spin-unrestricted DFT).

The Pt(111) surface was modeled as a periodic slab with four Pt layers, each containing 9 metal atoms in the unit cell $(3 \times 3$ cells $)$. The bottom two Pt layers were frozen in the crystal positions using the experimental equilibrium value of the bulk Pt lattice constant $(2.775 \AA)$, whereas the top two layers were free to relax along with the adsorbed species (for simplicity, this surface relaxation is not shown explicitly in the images of Fig. 1 to be discussed in the next section).

The solvation calculations used the approach for periodic slabs developed recently by Sha et $a .^{7}{ }^{7}$ This solvation (employing a continuum model $^{23,24}$ ) was calculated using the adaptive Poisson-Boltzmann solver (APBS) continuum method. ${ }^{25,34}$ The reaction pathways were determined using the Nudged Elastic Band (NEB) method, ${ }^{26-28}$ where the solvation effects were included a posteriori for each point along the path. In changing the dielectric constant of the medium $(\varepsilon)$, we calculated only the electrostatic polarization term, assuming that cavitation and dispersion/repulsion contributions to the solvation energy do not change. ${ }^{23,24}$ We considered variations in $\varepsilon$ from 78 (appropriate for water under standard conditions) to 1 (vacuum). The APBS continuum approach for modeling solvation assumes that the dielectric interface between a solvent and solute is a sharp contact surface defined by the van der Waals envelope with a probe solvent molecule. Here the continuum medium outside the solute is assumed to have the same dielectric constant up to the contact point.
OER steps

(A) $\mathrm{O}_{2}$ desorption

$\mathrm{O}_{2 \text { (ads) }} \rightarrow \mathrm{O}_{2(\mathrm{sol})}$

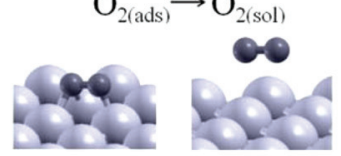

(B) $\mathrm{O}_{2}$ formation

$2 \mathrm{O}_{\text {(ads) }} \rightarrow \mathrm{O}_{2 \text { (ads) }}$

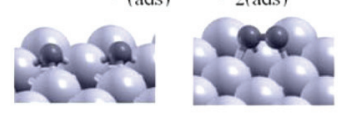

(C) $\mathrm{OH}$ dissociation

$\mathrm{OH}_{\text {(ads) }} \rightarrow \mathrm{O}_{\text {(ads) }}+\mathrm{H}_{\text {(ads) }}$

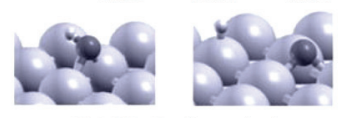

(D) $\mathrm{H}_{2} \mathrm{O}$ dissociation

$\mathrm{H}_{2} \mathrm{O}_{\text {(ads) }} \rightarrow \mathrm{OH}_{\text {(ads) }}+\mathrm{H}_{\text {(ads) }}$

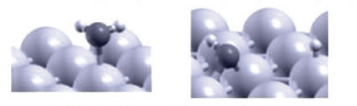

(E) $\mathrm{OOH}$ dissociation

$\mathrm{OOH}_{\text {(ads) }} \longrightarrow \mathrm{O}_{2 \text { (ads) }}+\mathrm{H}_{\text {(ads) }}$

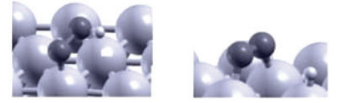

(F) $\mathrm{OOH}$ formation

$\mathrm{OH}_{(\mathrm{ads})}+\mathrm{O}_{(\mathrm{ads})} \underset{\mathrm{OOH}_{(\mathrm{ads})}}{ }$

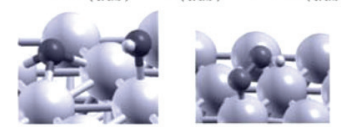

(G) $\mathrm{OH}$ disproportionation

$\mathrm{OH}_{\text {(ads) }}+\mathrm{OH}_{\text {(ads) }} \longrightarrow \mathrm{H}_{2} \mathrm{O}_{\text {(ads) }}+\mathrm{O}_{\text {(ads }}$

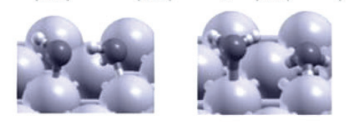

$\mathrm{OOH}_{\text {(ads) }}+\mathrm{H}_{(\mathrm{ads})} \rightarrow \mathrm{OH}_{\text {(ads) }}+\mathrm{OH}_{\text {(ads) }}$
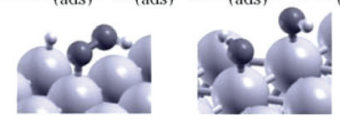

$(\mathrm{H}) \mathrm{OOH}$ dissociative hydrogenation
ORR steps

( I ) $\mathrm{O}_{2}$ adsorption

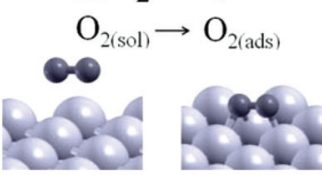

(L) $\mathrm{O}_{2}$ dissociation

$\mathrm{O}_{2 \text { (ads) }} \longrightarrow 2 \mathrm{O}_{\text {(ads }}$

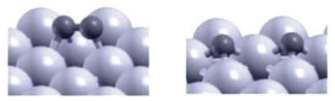

(M) $\mathrm{OH}$ formation

$\mathrm{O}_{(\mathrm{ads})}+\mathrm{H}_{\text {(ads) }} \rightarrow \mathrm{OH}_{(\mathrm{ads})}$

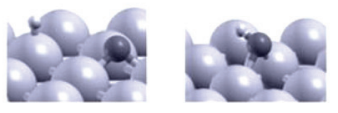

(N) $\mathrm{H}_{2} \mathrm{O}$ formation
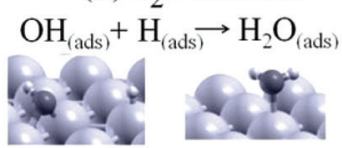

(O) $\mathrm{O}_{2}$ hydrogenation

$\mathrm{O}_{2 \text { (ads) }}+\mathrm{H}_{(\text {ads })} \rightarrow \mathrm{OOH}_{(\text {ads })}$

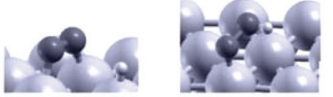

(P) $\mathrm{OOH}$ breaking $\mathrm{OOH}_{\text {(ads) }} \rightarrow \mathrm{OH}_{(\mathrm{ads})}+\mathrm{O}_{\text {(ads) }}$

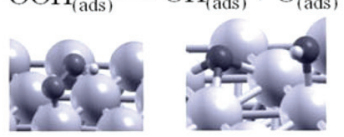

(Q) O hydration

$\mathrm{H}_{2} \mathrm{O}_{\text {(ads) }}+\mathrm{O}_{\text {(ads) }} \rightarrow \mathrm{OH}_{\text {(ads) }}+\mathrm{OH}_{(\text {ads })}$

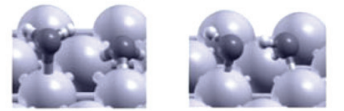

(R) $\mathrm{OH}$ associative deprotonation $\mathrm{OH}_{\text {(ads) }}+\mathrm{OH}_{\text {(ads) }} \rightarrow \mathrm{OOH}_{(\mathrm{ads})}+\mathrm{H}_{\text {(ads) }}$

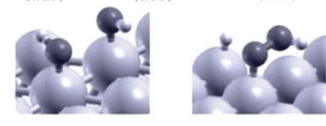

Fig. 1 Fundamental reaction steps of ORR/OER on $\mathrm{Pt}(111))^{7,8,20} \mathrm{H}, \mathrm{O}$, and Pt atoms in white, dark gray, and light gray color, respectively.

In these calculations we made several simplifying assumptions and approximations.

For example, we did not explicitly consider zero-point contributions to reaction energetics, and we did not evaluate Arrhenius pre-factors for energy barriers. While this is necessary for quantitative accuracy, we focus here on deriving general trends and the changes on the potential energy surface introduced by solvation.

Also we did not combine the Arrhenius prefactor for the rate determining step with the number of steps of the process into an the effective Arrhenius prefactor, ${ }^{3}$ as in the present case this does not amount to more than a factor of 2 . 


\section{Results and discussion}

In electrochemical cells, each process of the water energy cycle (1 and 2) is realized at a separate electrode. Specifically, water splitting occurs as oxygen evolution reaction (OER) and hydrogen evolution reaction (HER):

$$
\begin{gathered}
\text { OER (anode) }: \mathrm{H}_{2} \mathrm{O} \rightarrow \frac{1}{2} \mathrm{O}_{2}+2 \mathrm{H}^{+}+2 \mathrm{e}^{-} \\
\text {HER (cathode) }: 2 \mathrm{H}^{+}+2 \mathrm{e}^{-} \rightarrow \mathrm{H}_{2}
\end{gathered}
$$

while water formation involves the oxygen reduction reaction (ORR) and the hydrogen oxidation reaction (HOR):

$$
\begin{gathered}
\text { ORR (cathode) }: \frac{1}{2} \mathrm{O}_{2}+2 \mathrm{H}^{+}+2 \mathrm{e}^{-} \rightarrow \mathrm{H}_{2} \mathrm{O} \\
\text { HOR (anode) }: \mathrm{H}_{2} \rightarrow 2 \mathrm{H}^{+}+2 \mathrm{e}^{-}
\end{gathered}
$$

HER/HOR electrocatalysts are known to achieve fast kinetics under a wide range of conditions; ${ }^{29}$ however, OER/ORR catalysis is far slower with the mechanisms and the origin of their inefficiency is still quite uncertain. ${ }^{30,31}$

\section{A worked-out example: $\operatorname{Pt}(111)$}

To illustrate our approach for solvent optimization, we will focus on a specific catalyst: the fcc (111) platinum surface. ${ }^{32,33}$ The Pt(111) surface as an ORR catalyst has been investigated thoroughly, ${ }^{7,16}$ whereas Pt is well known to be a not good OER catalyst: experimentally, PtOx species develop on Pt surfaces under OER conditions when increasing the electrode potential above $\approx 1 \mathrm{~V}$, and OER eventually takes place with a significant over-potential on these oxidized surfaces. ${ }^{32,33}$ We selected Pt(111) here because rather thorough investigations of the ORR mechanistic steps as a function of the solvent dielectric constant have already been published, ${ }^{7,16}$ providing the framework to conduct the present analysis.

Fig. 1 shows the reaction steps involved in OER and ORR on $\operatorname{Pt}(111)$. We start with the reaction path energetics for ORR on Pt(111) calculated using PBE density-functional theory (DFT) calculations for the gas phase and for water using the APBS continuum model based on the Poisson-Boltzmann approximation. ${ }^{7,8}$ Recently ${ }^{16}$ we extended these energetics to solvents with various dielectric constants, using the same continuum model. ${ }^{34}$ As described in section 2, electrostatic polarization terms are included as the dielectric constant of the medium, $\varepsilon$, which is changed from 78 (appropriate for water under standard conditions) to 1 (vacuum), assuming constant cavitation and dispersion/repulsion contributions.

For simplicity, in Fig. 1 it is assumed that during water formation/oxidation reactions the metal support maintains the same basic atomistic structure, thus ignoring surface reconstruction and collective rearrangements involving adsorbates that may occur in the high-coverage régime. ${ }^{35}$ Also, coverage effects on reaction energetics are not investigated. As discussed above, we take the $\mathrm{Pt}(111)$ surface as an example of an electrode catalyst because a computational mechanistic investigation as a function of the medium polarizability is available. ${ }^{16}$ However, our approach of deriving mechanistic insight from tuning solvent properties should be relevant for a variety of surfaces. ${ }^{36}$ The picture provided here is a paradigmatic analysis, illustrating the application and the potential of the present approach, whose major goal is to provide a general framework for understanding and designing ORR/OER catalyst systems independent of the details of the catalyst and computational approach. ${ }^{36}$

The ORR/OER energetics with various values of the solvent dielectric constant ranging from pure water $(\varepsilon=78)$ to vacuum $(\varepsilon=1)$ as derived from calculations ${ }^{7,8,16}$ are reported in Table 1, while Fig. 2 shows the energy barriers as functions of the inverse of the solvent dielectric constant for each step considered in Fig. 1 and Table 1.

Charged species such as hydronium or hydroxyl ions are not included in Fig. 1, 2 and Table 1. However, the energetics of adsorption/desorption of $\mathrm{H}$ and $\mathrm{OH}$ species on the $\mathrm{Pt}(111)$ surface is known from the experiment and can be included into the modelling at various levels of accuracy. For example, the transfer of a hydrogen atom adsorbed on $\mathrm{Pt}(111), \mathrm{H}_{\mathrm{ad}}$, into the solution as a hydronium ion and the simultaneous release of an electron into the Pt electrode:

$$
\mathrm{H}_{\mathrm{ad}} \rightarrow \mathrm{H}_{\text {solv }}^{+}+\mathrm{e}^{-}
$$

corresponds to a standard free energy difference of $+0.40 \mathrm{eV},{ }^{37}$ implying e.g. that $\mathrm{H}_{\mathrm{ad}}$ is not present on the

Table 1 Energetics of the fundamental reaction steps of ORR/OER on $\mathrm{Pt}(111){ }^{7,8,16}$ ORR reaction energy differences ( $\Delta E^{\mathrm{ORR}}$ ) and reaction energy barriers for ORR and OER: $E_{\mathrm{b}}^{\mathrm{ORR}}$ and $E_{\mathrm{b}}^{\mathrm{OER}}$, respectively, are reported for various values of the solvent dielectric constant ranging from vacuum, $\varepsilon=1$, to water, $\varepsilon=78$. Note that energy differences correspond to the ORR, and their sign must be changed when reversing the direction of the reaction coordinate in the OER

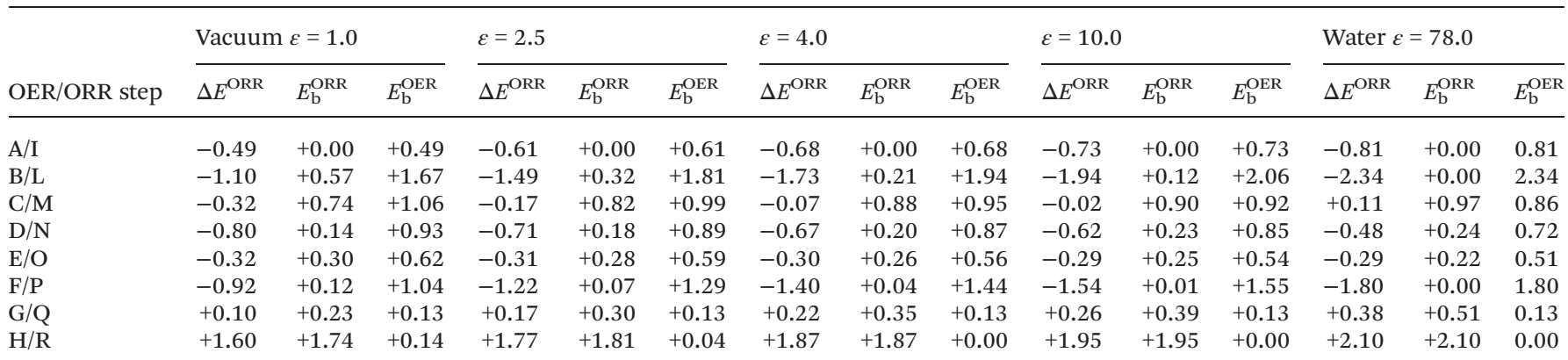


(a) OER steps

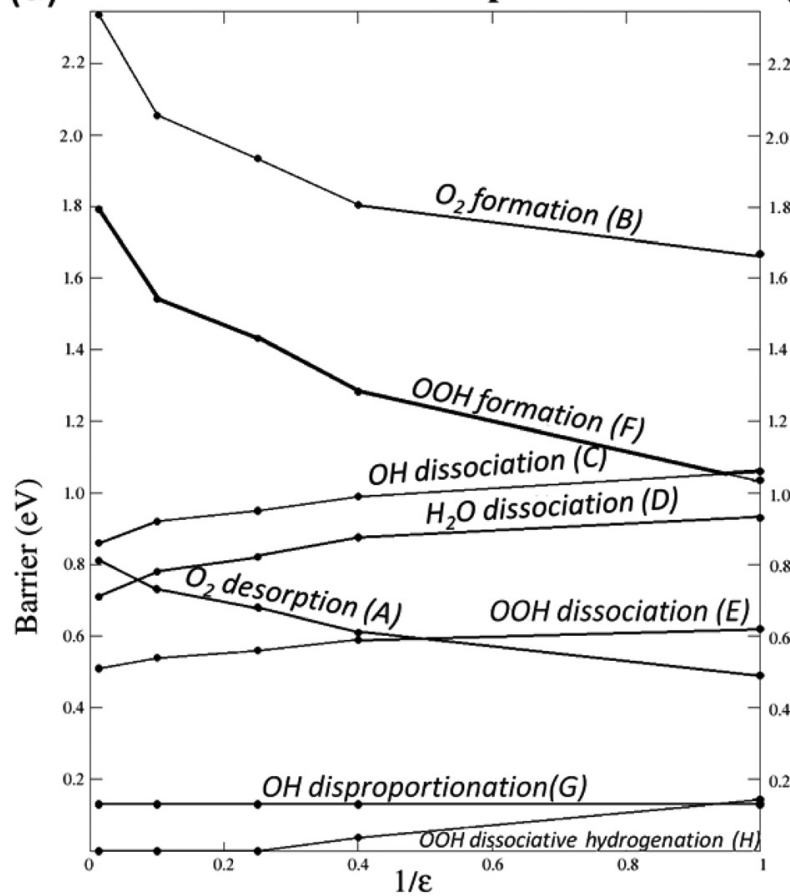

(b) ORR steps

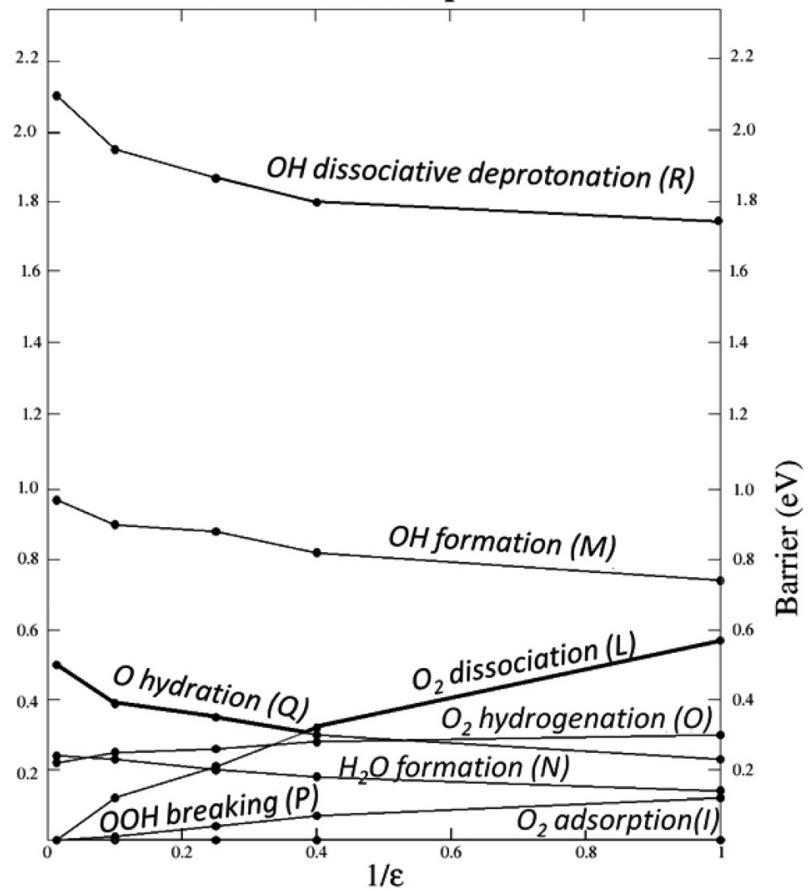

Fig. 2 Predicted energy barriers for the individual reaction steps of the (a) OER and (b) ORR on Pt(111) as a function of the inverse of the dielectric constant of the solvent $\varepsilon .{ }^{16}$ The nomenclature of reaction steps is the same as in Fig. 1 . The rate determining steps at various values of $(1 / \varepsilon)$ are highlighted in bold.

surface under OER conditions. As shown previously, ${ }^{3-6}$ the effect of the electrode potential on the reaction energies for processes involving hydrogen desorption (or adsorption) can be included approximately by shifting by the magnitude $+e V$ (with $e$ being the unit charge and $V$ the potential) the energy of products such as in eqn (7). A more complete investigation of the effect of electrode potential on the energy profile (barriers) is not straightforward but has been considered in the DFT calculations for some steps of the ORR on $\mathrm{Pt}(111){ }^{9}$

Before illustrating our proposal, we digress about OER over $\mathrm{Pt}(111)$. Pt(111) per se is not a good OER catalyst: rather, oxides such as $\mathrm{IrO}_{2}$ or $\mathrm{RuO}_{2}$ are efficient systems. ${ }^{38}$ However, we will show hereafter that understanding why Pt(111) is not a good catalyst although it is an excellent ORR catalyst and can be useful for a rational design of OER catalytic systems. Moreover, we show that studying the dependence of Pt(111) OER activity upon the solvent dielectric constant provides useful insights.

From Table 1, the main issue of achieving efficient OER is singled out as the difficulty in forming and stabilizing the $\mathrm{O}-\mathrm{O}$ bond over the catalyst surface. ${ }^{3}$ Three pathways have been established to form an $\mathrm{O}-\mathrm{O}$ bond:

1. Direct OO formation. Here two adsorbed $\mathrm{O}$ atoms on the surface are combined directly:

$$
\mathrm{O}_{2} \text { formation: } \mathrm{O}_{\mathrm{ad}}+\mathrm{O}_{\mathrm{ad}} \rightarrow \mathrm{O}_{2 \mathrm{ad}} \quad \text { (step B) }
$$

We find that the barrier for this is $1.67 \mathrm{eV}$ in the gas phase which becomes $2.34 \mathrm{eV}$ in water.

2. $\mathrm{H}$-assisted $\mathrm{OO}$ formation. Here $\mathrm{O}_{2}$ formation involves two steps. First $\mathrm{O}_{\mathrm{ad}}$ and $\mathrm{OH}_{\mathrm{ad}}$ are combined to form $\mathrm{HOO}_{\mathrm{ad}}$ :

$$
\text { OOH formation: } \mathrm{O}_{\mathrm{ad}}+\mathrm{OH}_{\mathrm{ad}} \rightarrow \mathrm{HOO}_{\mathrm{ad}} \quad \text { (step F) }
$$

which is followed by decomposition of the $\mathrm{HOO}_{\mathrm{ad}}$ to form gas phase $\mathrm{O}_{2}$ and $\mathrm{H}_{\mathrm{ad}}$,

$$
\text { OOH dissociation: } \mathrm{OOH}_{\mathrm{ad}} \rightarrow \mathrm{O}_{2 \mathrm{ad}}+\mathrm{H}_{\mathrm{ad}} \quad \text { (step E) }
$$

We find that $\mathrm{OOH}_{\mathrm{ad}}$ formation is thermodynamically disfavored by $0.9 \mathrm{eV}$ in the gas phase which becomes $1.8 \mathrm{eV}$ in water, with the corresponding barriers of $1.04 \mathrm{eV}$ in the gas phase and $1.8 \mathrm{eV}$ in water. Although still high, these values are smaller than for the direct $\mathrm{O}_{2}$ formation step B. Moreover, as regards the main premise in this work, we note that solvation in water provides a sizeable contribution to the energy barrier: $0.76 \mathrm{eV}$, so that reducing the polarizability of the medium should improve the viability of this pathway.

3. Peroxide mechanism. There is also a third possibility: the formation of hydrogen peroxide from two hydroxyls:

$$
\text { Peroxide formation: } \mathrm{OH}_{\mathrm{ad}}+\mathrm{OH}_{\mathrm{ad}} \rightarrow \mathrm{H}_{2} \mathrm{O}_{2 \mathrm{ad}}
$$


which subsequently loses the $\mathrm{H}$ atoms one by one to form $\mathrm{O}_{2}$. However, this is suppressed by the much more favorable $\mathrm{OH}$ disproportionation:

OH disproportionation: $\mathrm{OH}_{\mathrm{ad}}+\mathrm{OH}_{\mathrm{ad}} \rightarrow \mathrm{O}_{\mathrm{ad}}+\mathrm{H}_{2} \mathrm{O}_{\mathrm{ad}} \quad$ (step G)

Both steps $(8,9)$ are highly endothermic, and both become even more endothermic in a strongly polarizable, high-dielectric-constant solvent such as water because the solvation energies of adsorbed dioxygen $\mathrm{O}_{2 \mathrm{ad}}$ or hydroperoxyl radical $\mathrm{OOH}_{\mathrm{ad}}$ are smaller than those of the adsorbed oxygen atom or hydroxyl $\mathrm{OH}_{\mathrm{ad}}$ species. Moreover, as observed above solvation in water gives a further sizeable contribution to the energy barrier of the $\mathrm{OOH}_{\mathrm{ad}}$ formation step: $0.76 \mathrm{eV}$. It is also important to underline that applying a potential does not strongly affect such energy differences so that this issue of stabilizing the $\mathrm{O}-\mathrm{O}$ bond is expected to hold independent of the value of the bias potential. ${ }^{39}$

Another crucial point is that the barrier to dissociation of adsorbed $\mathrm{O}_{2}$ is nearly zero in water so that any $\mathrm{O}_{2}$ produced might re-react to form $\mathrm{O}_{\mathrm{ad}}+\mathrm{O}_{\mathrm{ad}}$ (interestingly this barrier can also become negligible in small clusters, see e.g. ref. 50). But this barrier increases for less polarizable media, making this process potentially less important. A similar reasoning holds for $\mathrm{OOH}$ dissociation, whose kinetic stability is also important to stabilize the path leading to $\mathrm{O}_{2}$.

Coming explicitly to the mechanisms which may take place on $\mathrm{Pt}(111)$, for ORR at $1 / \varepsilon<0.38$, the steps are: step L to dissociate $\mathrm{O}_{2}$ to form $\mathrm{O}_{\mathrm{ad}}$, step Q to hydrolyze $\mathrm{O}_{\mathrm{ad}}$ to $\mathrm{OH}_{\mathrm{ad}}$, step $\mathrm{N}$ to form $\mathrm{H}_{2} \mathrm{O}$ by protonating $\mathrm{OH}_{\mathrm{ad}}$, leading to step $\mathrm{Q}$ as the rate determining step (RDS). For $1 / \varepsilon>0.38$, step $\mathrm{O}$ hydrogenation of $\mathrm{O}_{2}$ to form $\mathrm{OOH}_{\mathrm{ad}}$ followed by step $\mathrm{P}$ to form $\mathrm{O}_{\mathrm{ad}}$ and $\mathrm{OH}_{\mathrm{ad}}$ becomes more favorable than step $\mathrm{L}$, but the RDS remains step $\mathrm{Q}$ until $1 / \varepsilon>0.55$ where step $\mathrm{O}$ becomes the RDS. Thus the highest rate should be for $1 / \varepsilon \sim 0.55$.

For OER at all $1 / \varepsilon \gtrsim 0.95$, the steps are step $\mathrm{D}$ to form $\mathrm{OH}_{\mathrm{ad}}$ and $\mathrm{H}_{\mathrm{ad}}$ from absorbed $\mathrm{H}_{2} \mathrm{O}$, followed by step $\mathrm{G}$ to form $\mathrm{O}_{\mathrm{ad}}+$ $\mathrm{H}_{2} \mathrm{O}_{\mathrm{ad}}$ from two hydroxyls, followed by step $\mathrm{F}$ to combine $\mathrm{O}_{\mathrm{ad}}$ and $\mathrm{OH}_{\mathrm{ad}}$ to form $\mathrm{OOH}_{\mathrm{ad}}$, followed by step $\mathrm{E}$ to form $\mathrm{O}_{2 \mathrm{ad}}$, followed by step A to release free $\mathrm{O}_{2}$ from the surface. The overall RDS is step F, but never step B. However, for $1 / \varepsilon<0.95$, step C to convert $\mathrm{OH}_{\mathrm{ad}}$ to $\mathrm{O}_{\mathrm{ad}}+\mathrm{H}_{\mathrm{ad}}$ has a lower barrier, preventing step $\mathrm{F}$. In this case the $\mathrm{O}_{\mathrm{ad}}$ concentration would build up until there are no sites to accommodate step $\mathrm{C}$, at which point step $\mathrm{F}$ could again occur. Thus step $\mathrm{F}$ is again the RDS, now for all $\varepsilon$.

In short, a candidate OER path over a $\mathrm{Pt}(111)$ catalyst surface is the following:

$$
\mathrm{H}_{2} \mathrm{O} \text { dissociation: } \mathrm{H}_{2} \mathrm{O}_{\mathrm{ad}} \rightarrow \mathrm{HO}_{\mathrm{ad}}+\mathrm{H}_{\mathrm{ad}} \quad \text { (step D) }
$$

OH disproportionation: $\mathrm{OH}_{\mathrm{ad}}+\mathrm{OH}_{\mathrm{ad}} \rightarrow \mathrm{O}_{\mathrm{ad}}+\mathrm{H}_{2} \mathrm{O}_{\mathrm{ad}}$

OOH formation: $\mathrm{O}_{\mathrm{ad}}+\mathrm{OH}_{\mathrm{ad}} \rightarrow \mathrm{HOO}_{\mathrm{ad}} \quad($ step F)

$$
\begin{gathered}
\text { OOH dissociation: } \mathrm{HOO}_{\mathrm{ad}} \rightarrow \mathrm{O}_{2 \mathrm{ad}}+\mathrm{H}_{\mathrm{ad}} \quad \text { (step E) } \\
\text { H dissolution: } \mathrm{H}_{\mathrm{ads}} \rightarrow \mathrm{H}_{\text {solv }}^{+}+\mathrm{e}^{-}
\end{gathered}
$$

The possible reaction pathways for ORR and OER are pictorially shown in Fig. 3 for convenience of the reader.

The conclusion of this digression is that tuning the medium polarizability can appreciably modify the OER electrocatalytic activity of a given system.

\section{The dielectric sensitivity strategy for improving OER and ORR catalysts}

The above discussion and Fig. 2, showing that the energy barriers of the individual ORR and OER reaction steps exhibit a quite diverse behavior as a function of the inverse of the solvent dielectric constant, lay the basis for the proposed dielectric sensitivity strategy for improving OER and ORR catalysts.

Thus for some new catalyst system, we propose that the first step in improving the catalyst is to measure experimentally how the overall ORR/OER depend on the solvent dielectric constant, say for two or three solvent cases, and compare with Fig. 2. Based on the slope one can identify which regime this catalyst is and use this information to identify the likely RDS. Then focus on this hypothesized RDS making variations in the catalyst that are expected to decrease the barrier.

Thus for the case of ORR on $\operatorname{Pt}(111)$ in the range of large $\varepsilon$, the derivatives of the energy barriers with respect to $(1 / \varepsilon)$ range from

- a negligible value - steps I,P

- to small positive or negative values - steps $\mathrm{O}$ and $\mathrm{N}$, respectively

- up to significantly positive values for step L or

- significantly negative values for steps M, Q, and R.

Similarly for OER on $\operatorname{Pt}(111)$, the derivatives of the energy barriers with respect to $(1 / \varepsilon)$ range

- from negligible values - steps $\mathrm{G}$, and $\mathrm{H}$

- to small positive values - step E

- up to significantly positive values for steps D and C or

- significantly negative values for steps A, B, and F.

These observations provide the basis for identifying the RDS.

The reasons for such a diverse response to the perturbation produced by a change in the solvent polarizability arise because the ORR/OER individual steps exhibit a diverse range of electron redistributions during the reactions: ranging from creation or disappearance of strong hydrogen bonds and/or substantial variations in the polarity of reactants and transition states, to minor changes and adjustments. The key kinetic quantity that is sensitive to the solvent medium is the difference in polarity between reactants and transition states .

We should stress that this strong diversity can be expected in general for such reactions, because of the wide range of electron redistributions associated with the reaction steps involving $\mathrm{O}$ - and $\mathrm{H}$-species is intrinsic to OER/ORR. Thus it should be possible to use the dielectric constant discriminate 
(D)

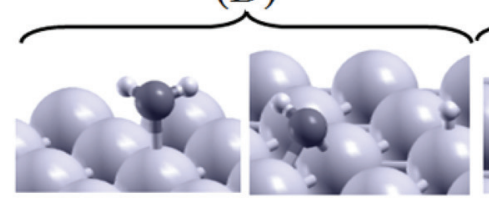

OER on Pt(111)

\author{
(G)
}

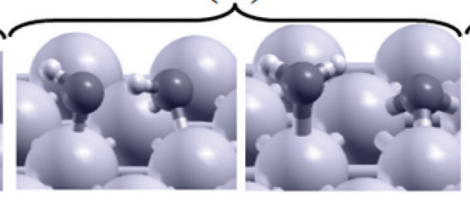

(F)

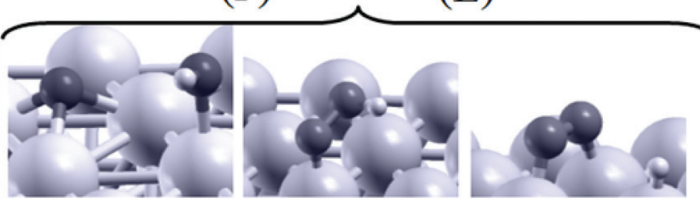

\section{ORR on Pt(111) High $\varepsilon$}

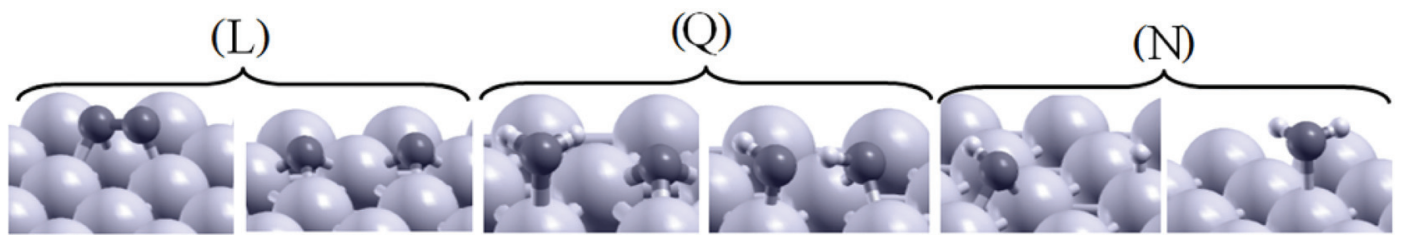

\section{ORR on Pt(111) Low ع}

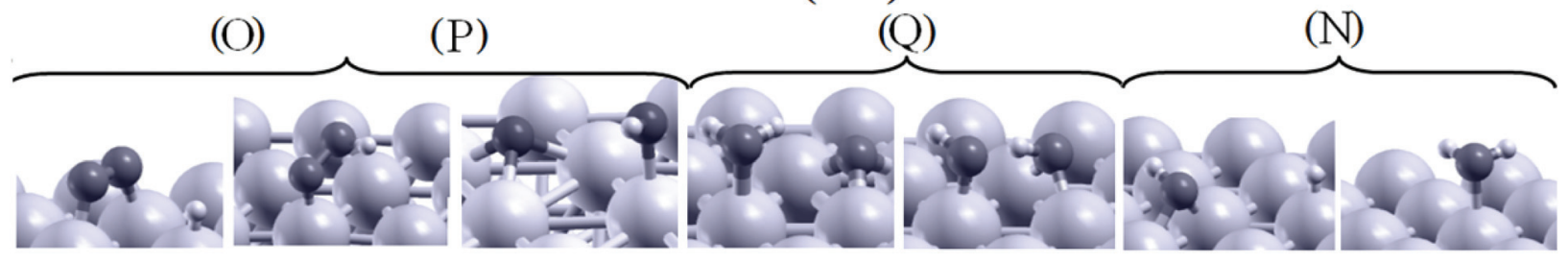

Fig. 3 Pictorial illustration of the possible reaction pathways for OER and ORR on $\mathrm{Pt}(111)$.

the OER/ORR rate determining steps among the possible reaction mechanisms.

It should be noted that the above general analysis made several assumptions to simplify the discussion.

1. In Table 1 and Fig. 2 we included only electronic energy contributions to reaction barriers whereas entropy must be included to get rates, which depend on free energy. We have developed a general procedure for doing this from molecular dynamics in an explicit solvent, ${ }^{40-42}$ but not for an implicit solvent.

2. We focused our analysis on the energetics at the catalyst site, but changing the solvent medium also affects transport phenomena and the solvent double layer, which must be taken into account for quantitative modeling. Interestingly, statistical mechanics-based models describing the double layered structure and associated electric permittivity variation at the vicinity of the catalyst ${ }^{43}$ could be coupled to the present calculations by defining a space-dependent solvent dielectric constant.

3 . The reaction paths can change under environmental conditions (such as the solvent polarizability). For example, the mechanism of HER on Pt(111) changes from a hydroniumbased one at low $\mathrm{pH}$ to a water-based one at high $\mathrm{pH} .{ }^{29}$ Even without a change in the overall mechanism, the rate determining step can change as a function of the solvent dielectric constant simply because the barrier of the step that is initially rate determining becomes smaller than that of another step, which then becomes rate determining. This is the reason why the overall kinetic rate is expected to exhibit a maximum as a function of the solvent dielectric constant, in tune with the Sabatier principle of catalysis. ${ }^{44}$ Thus the best strategy is to study the derivative of kinetic rate via small perturbations in the solvent dielectric constant as a function of $\varepsilon$.

4. Our analysis is valid only for non-reactive solvents.

Given these predictions, it will be interesting to find experimental corroboration of our approach. Mixing water with other solvents is a simple and effective way to tune the dielectric constant of the medium (another possibility is discussed below $\left.^{16}\right)$. Consider for example tetrahydrofuran (THF) - or $\left(\mathrm{CH}_{2}\right)_{4} \mathrm{O}-$ as a co-solvent. ${ }^{45}$ THF has a low dielectric constant of 7.8 under standard conditions, and is fully miscible with water. A mixture of THF-water in a molar ratio 80:20 has a dielectric constant ${ }^{46}$ of $\approx 10$, corresponding to the second leftmost points in Fig. 2(a,b). Mixing water with THF in other ratios or with other solvents can produce all the points in Table 1 and Fig. 2.

Experimental investigations of the OER/ORR kinetics as a function of solvent composition have not yet been reported. However, recent experiments show an acceleration of ORR kinetics upon changing the Nafion concentration in the catalyst. $^{51}$ This may be interpreted as a water confinement effect leading to a reduction in the medium dielectric constant, and thus may be explained by the present analysis. Moreover, a study along the line of solvent mixing has been explored in the context of HER photocatalysis, for example ref. 47 that investigated a THF- $\mathrm{H}_{2} \mathrm{O}$ mixture with a 80:20 molar ratio and showed that it accelerates photocatalytic HER kinetics by $43 \%$ 
with respect to an equimolar 50:50 solution. This acceleration was attributed $^{47}$ to the influence of the solvent on optical excitation energies, which in photochemistry goes under the name of solvatochromic effect. ${ }^{48,49}$ The solvatochromic effect arises from the difference in the dipole moment of ground and excited electronic states, which produces a differential response to a change in solvent polarity. Indeed, an analogy can be drawn between the ground/excited state pair in solvatochromism and the reactant/transition-state pair in the rate determining step of catalytic processes - in both cases the response of the system to a change in the dielectric properties of the solvent is determined by the differential polarities of the two states. However we speculate that the kinetic acceleration reported in ref. 47 may actually arise from the effect of the solvent dielectric constant $\varepsilon$ on the HER reaction energy barrier, rather than solvatochromism. In this case the experiment implies a derivative of the rate determining step energy barrier with respect to $(1 / \varepsilon)$ of $-0.3 \mathrm{eV}$. If so, this result could be used help test the catalytic reaction mechanism.

\section{Conclusions}

In summary, we show here that the kinetics and mechanism of water oxidation/formation reactions can be modified drastically by changing the solvent polarizability. Taking the Pt(111) surface as a model example for illustrative purposes, we identify the origin of its ineffectiveness as an OER catalyst as the difficulty in forming and stabilizing the $\mathrm{O}-\mathrm{O}$ bond over the catalyst surface, which becomes more acute in a strongly polarizable high-dielectric-constant solvent such as water. We show that this deficiency could be partially overcome by reducing the medium polarizability.

Apart from the analysis of such specific examples, we suggest that our solvent polarization optimization approach provides a way to extract and validate the mechanistic understanding to help suggest improved materials. Our experimental design approach is based on the dramatically different dependence of ORR/OER energy barriers upon solvation (see Fig. 2). We suggest that this approach of combining experimental/ theoretical studies of the electrochemical water-based energy cycle as a function of environmental variables can provide an effective tool to derive the mechanistic information needed for achieving tailoring ORR/OER catalyst electrodes and materials sufficiently to make a practical water energy cycle a reality, thus facilitating the rise of a much needed hydrogen economy.

\section{Acknowledgements}

This work was supported by the National Science Foundation (CBET-1067848) and by a Short-Term Mission (STM) funded by Italian Consiglio Nazionale delle Ricerche.

\section{References}

1 S. Dunn, Int. J. Hydrogen Energy, 2002, 27, 235-264.

2 M. K. Debe, Nature, 2012, 486, 43-51.
3 J. K. Norskov, J. Rossmeisl, A. Logadottir, L. Lindqvist, J. R. Kitchin, T. Bligaard and H. Jonsson, J. Phys. Chem. B, 2004, 108, 17886-17892.

4 A. U. Nilekar and M. Mavrikakis, Surf. Sci., 2008, 602, L89L94.

5 J. Rossmeils and J. K. Norskov, Surf. Sci., 2008, 602, 23372338.

6 J. A. Keith and T. Jacob, Angew. Chem., Int. Ed., 2010, 49, 9521-9525.

7 Y. Sha, T. H. Yu, B. V. Merinov, P. Shirvanian and W. A. Goddard, J. Phys. Chem. Lett., 2011, 2, 572-576.

8 Y. Sha, T. H. Yu, Y. Liu, B. V. Merinov and W. A. Goddard, J. Phys. Chem. C, 2010, 1, 856-861.

9 Y. Sha, T. H. Yu, B. V. Merinov and W. A. Goddard, J. Phys. Chem. C, 2012, 116, 6166-6173.

10 T. H. Yu, Y. Sha, B. V. Merinov and W. A. Goddard, J. Phys. Chem. C, 2010, 114, 11527-11533.

11 T. H. Yu, T. Hofmann, Y. Sha, B. V. Merinov, D. J. Myers, C. Heske and W. A. Goddard, J. Phys. Chem. C, 2013, 117, 26598-26607.

12 Y. Sha, T. H. Yu, B. V. Merinov, P. Shirvanian and W. A. Goddard, J. Phys. Chem. C, 2012, 116, 21334-21342.

13 N. M. Markovic, Nat. Mater., 2014, 12, 101-102.

14 M. Zhang, M. de Respinis and H. Frei, Nat. Chem., 2014, 6, 362-367.

15 T. H. Yu, Y. Sha, W.-G. Liu, B. V. Merinov, P. Shirvanian and W. A. Goddard III, J. Am. Chem. Soc., 2011, 133, 1985719863.

16 A. Fortunelli, W. A. Goddard, Y. Sha, T. H. Yu, L. Sementa, G. Barcaro and O. Andreussi, Angew. Chem., Int. Ed., 2014, 53, 6669-6672.

17 J. Perdew, K. Burke and M. Ernzerhof, Phys. Rev. Lett., 1996, 77, 3865-3868.

18 P. Schultz, SeqQuest code project; Sandia National Laboratories: Albuquerque, NM (http://www.cs.sandia.gov/ $\sim$ paschul/Quest/).

19 C. F. Melius and W. A. Goddard, Phys. Rev. A, 1974, 10, 1528-1540.

20 C. F. Melius, B. D. Olafson and W. A. Goddard, Chem. Phys. Lett., 1974, 28, 457-462.

21 A. Redondo, W. A. Goddard and T. C. Mcgill, Phys. Rev. B: Solid State, 1977, 15, 5038-5048.

22 D. R. Hamann, Phys. Rev. B: Condens. Matter, 1989, 40, 2980-2987.

23 J. Tomasi and M. Persico, Chem. Rev., 1994, 94, 2027-2094.

24 C. Cramer and D. Truhlar, Chem. Rev., 1999, 99, 2161-2200.

25 D. J. Tannor, B. Marten, R. Murphy, R. A. Friesner, D. Sitkoff, A. Nicholls, M. Ringnalda, W. A. Goddard and B. Honig, J. Am. Chem. Soc., 1994, 116, 11875-11882.

26 G. Mills and H. Jonsson, Phys. Rev. Lett., 1994, 72, 1124-1127.

27 G. Mills, H. Jonsson and G. K. Schenter, Surf. Sci., 1995, 324, 305-337.

28 G. Henkelman, B. P. Uberuaga and H. Jonsson, J. Chem. Phys., 2000, 113, 9901-9904.

29 D. Strmcnik, M. Uchimura, C. Wang, R. Subbaraman, N. Danilovic, D. van der Vliet, A. P. Paulikas, 
V. R. Stamenkovic and N. M. Markovic, Nat. Chem., 2013, 5, 300-306.

30 Y. H. Fang and Z. P. Liu, J. Am. Chem. Soc., 2010, 132, 18214-18222.

31 N. Nepal and S. Das, Angew. Chem., Int. Ed., 2013, 52, 72247227.

32 B. E. Conway and T. C. Liu, Langmuir, 1990, 6, 268-276.

33 A. M. Gomez-Marin, J. Clavilier and J. M. Feliu, J. Electroanal. Chem., 2013, 688, 360-370.

34 N. Baker, D. Sept, S. Joseph, M. Holst and J. McCammon, Proc. Natl. Acad. Sci. U. S. A., 2001, 98, 10037-10041.

35 At high electrode potentials the formation of Pt oxides is known to occur in OER. ${ }^{33}$

36 It is not necessarily associated with a specific computational model. For example, the approach employed in ref. 16 used a few simplifications, such as the observations that the simulations did not explicitly take into account that the solvent dielectric constant at the interface may be different from its bulk value and that hydrogen bonding may not be adequately described by continuum models. Thus fully atomistic simulations may be needed to achieve quantitative accuracy. Indeed ref. 16 did not account for pathways in which charged species such as hydronium or hydroxyl ions take part in the reaction and neither considered surface reconstructions and/or collective adsorbate rearrangements, nor high-coverage effects on reaction energy profiles. Finally, the DFT exchange and correlation functional used here is accurate for valence structures and energies, but does not include dispersion interactions that might change some quantitative details.

37 F. Eisert and A. Rosén, Surf. Sci., 1997, 377-379, 759-764.
38 L. F. L. Oliveira, S. Laref, E. Mayousse, C. Jallut and A. A. Franco, Phys. Chem. Chem. Phys., 2012, 14, 1021510224.

39 Our DFT calculations find that charge transfer from the metal surface to the adsorbed $\mathrm{O}_{2}$ is small, but it may increase for other exchange-correlation DFT functionals.

40 T. A. Pascal and W. A. Goddard, J. Phys. Chem. B, 2014, 118, 5943-5956.

41 T. A. Pascal and W. A. Goddard, J. Phys. Chem. B, 2012, 116, 13905-13912.

42 T. A. Pascal, W. A. Goddard, P. K. Maiti and N. Videhi, J. Phys. Chem. B, 2012, 116, 12159-12167.

43 M. A. Quiroga, K. H. Xue, T. K. Nguyen, M. Tułodziecki, H. Huang and A. A. Franco, J. Electrochem. Soc., 2014, 161, E3302-E3310.

44 P. Sabatier, Ber. Dtsch. Chem. Ges., 1911, 44, 1984-2001.

45 We choose THF because it has been considered in ref. 47, but, to prevent formation of radicals, 2-2'-5-5'-tetramethylTHF (a non-reactive solvent) rather than THF should perhaps be used in OER studies.

46 F. E. Critchfield, J. A. Gibson and J. L. Hall, J. Am. Chem. Soc., 1953, 75, 6044-6045.

47 E. D. Cline, S. E. Adamson and S. Bernhard, Inorg. Chem., 2008, 47, 10378.

48 M. Cossi and V. Barone, J. Chem. Phys., 2000, 112, 24272435.

49 A. Marini, A. Munoz-Losa, A. Biancardi and B. Mennucci, J. Phys. Chem. B, 2010, 114, 17128-17135.

50 P. C. Jennings, H. A. Aleksandrov, K. M. Neyman and R. L. Johnston, Nanoscale, 2014, 6, 1153-1165.

51 J. Spedera and A. Zana, Matthias Arenz, private communication. 